\title{
Curt Nimuendaju, Etnografia Ticuna e fronteira Amazônica ${ }^{1}$
}

Priscila Faulhaber (Mast)

Junho de 2010

Este trabalho pretende dar uma contribuição para a discussão sobre o conceito de fronteira focalizando Curt Nimuendaju e a produção da etnografia Ticuna, índios que vivem em área de fronteira entre Brasil, Colômbia e Peru. Circunscreve tal contribuição no exame crítico do contexto da definição dos estudos de área pela antropologia produzida nos Estados Unidos durante a segunda Guerra Mundial, Trata-se, antes que se ater ao objeto do registro etnográfico da relação com os índios Ticuna - já estabelecido em outras ocasiões e que deverá ser objeto de outros trabalhos - considerar como a produção de tal etnografia é relevante para pensar a fronteira em termos antropológicos: A etnografia estará presente, todavia, no decorrer do texto à medida que se incorpora tal prática na leitura, exame de documentos escritos, procurando escutar as vozes dos autores em questão e identificar aspectos de suas interlocuções que se tornam interessantes para a análise antropológica.

Curt Nimuendaju produziu sua monografia sobre os índios Ticuna (Nimuendaju, 1952)., editada por Robert Lowie e publicada após a trágica morte do etnógrafo, entre os índios Ticuna, em dezembro de 1945. Lowie individualmente tinha grande admiração pelo trabalho de Nimuendaju e o considerava como um experiente colega. Naquele momento, no entanto, os trabalhos etnográficos de quem que não recebera treinamento acadêmico em universidades norte-americanas eram classificados, no campo antropológico, como não estruturados cientificamente. Tratava-se, entre outros motivos,

\footnotetext{
${ }^{1}$ Trabalho apresentado na 27ạ. Reunião Brasileira de Antropologia, realizada entre os dias $01 \mathrm{e}$ 04 de agosto de 2010, Belém, Pará, Brasil.
} 
de uma dificuldade de enquadramento, uma vez que tais etnografias mostravam realidades ambíguas, em outras palavras, fronteiriças, que não correspondiam às expectativas daqueles que viam os povos ameríndios - etnocentricamente - como entidades homogêneas.

Isto ocorria em uma situação especial de assimetria de poder entre estabelecimentos científicos do Sul e do Norte, quando, em meados do século XX, os Estados Unidos passaram a ocupar uma posição privilegiada na disputa pela hegemonia no campo cientifico, passando a deter controle sobre os processos de apropriação e disseminação do conhecimento. A dinâmica das trocas simbólicas criou uma cadeia de interdependências baseada em uma disputa por apropriações culturais. Tal dinâmica envolveu a criação de grupos de especialistas que manejavam um "fundo específico de representações simbólicas" (Elias, 1982:43), criando e estruturando domínios de conhecimento que poderiam ser enquadrados como "subcontinentes" como "América do Sul" ou regiões como a Amazônia, que despertava interesse por abrigar vasta floresta tropical. Tal compartimentalização do conhecimentto se produzia em circunstâncias específicas nas quais evidenciam-se as hierarquias implicadas na objetivação de conteúdos culturais e no estranhamento pelos autores de suas criações. Tais hierarquias condicionaram a divisão do trabalho, que implicava, em crescente diferenciação, como caracterizaria Simmel (1968),

Considerando tal visão hierárquica em uma crítica da "antropologia do colonialismo" (Pels, 1997, 2008), parte-se aqui da concepção que tal hierarquização constituiu, em tais circunstâncias, definições específicas de "estudos de área" como lugares da antropologia subjacentes a uma idéia homogeneizadora de "área" ou “região". Tal hierarquização foi reiterada por antropólogos que elaboravam sínteses teóricas com bases em descrições produzidas etnograficamente por aqueles que 
produziam seus trabalhos em um campo considerado como não completamente estruturado do ponto de vista dos padrões de ciência da época.

Os índios da floresta tropical na Amazônia tiveram, desde meados do século XX, significado especial para a antropologia dos Estados Unidos, cujo foco, até então centrava-se sobre os índios de seu próprio país. Este interesse se dava não apenas pelo exotismo e pela busca de nativos vivendo em um estado de "autenticidade" ou por motivos comparativos, mas por propósitos informados por interesses políticos: quando os antropólogos, em resposta ao "esforço de guerra", mobilizavam-se na luta contra o nazismo e o fascismo, o índio passou a ser visto como um símbolo da integração panamericana. Neste contexto, a antropologia na Amazônia constituía como uma "linha de frente" para a pesquisa antropológica, ou seja, como uma "fronteira científica".

\section{Estruturação de estabelecimentos e práticas científicas em tempos de guerra}

A ideia de projetos colaborativos como o Hanbook of South American Indians e o Instituto de Antropologia Social surgiu quando Julian Steward vislumbrou a possibilidade de recursos do congresso estadunidense para a cooperação com as repúblicas da América do Sul como parte da "política de boa vizinhança". A apropriação dos recursos para tais projetos foi viabilizada mediante conexão do Comitê Interdepartamental com o Departamento de Estado dos EUA.

Nas circunstâncias da guerra uma conjunção de fatores criaram condições favoráveis à obtenção de recursos para a divulgação de pesquisa antropológica na América do Sul e nas florestas tropicais. O Ethnogeographic Board (Comitê Etnogeográfico, doravante EB) foi criado em 1942 como uma organização especial para atender a demandas do "esforço de guerra". Este comitê foi estabelecido e apoiado por 
uma articulação do National Research Council do American Council of Learned Societies, o Social Sciences Research Council e a Smithsonian Institution. O EB era uma organização não governamental cujo propósitzo era criar uma rede de especialistas que pudesse fornecer em tempo hábil conhecimento especializado para o sucesso em tarefas geradas pela situação de guerra (Bennet, 1947:22). Era organizado como uma clearing house (escritório de conversão e compensação) para prover às agências militares mobilizadas pela guerra, informação de regiões específicas e dados pessoais obtidos mediante pesquisa direta.

Este comitê constituiu um veículo de institucionalização dos estudos regionais, os chamados "estudos de área". Inicialmente a divisão em áreas na antropologia obedecia a problemas de exibição através dos quais os continentes, regiões e áreas eram mapeados. Tal distribuição etnogeográfica associava elementos culturais no espaço de acordo com uma hierarquia científica que estabelecia padrões de difusão uniforme que convencionava centros que determinavam determinada "área cultural" em termos dos quais eram definidas periferias. Determinando que os traços mais largamente distribuídos seriam necessariamente os mais antigos, eram produzidas sequências de desenvolvimento temporais. Tratava-se um referencial conservador poligenista associado ao posicionamento socialista de Franz Boas que se opunha à determinação do conhecimento pelos propósitos da guerra. (Stocking, 1976:20). Nos anos entre guerras, o campo científico se dividiu entre os que defendiam tal isenção profissional e outros que advogavam a aplicabilidade científica. Durante a Segunda Grande Guerra, tal processo de institucionalização delineou uma "geografia do conhecimento" específica para a situação de guerra (Nugent, 2008:34), configurada para atender o desejo militar de impor controle e estabilidade em extensivos territórios previamente objeto da 
colonização européia que os EUA visavam incorporar, aproveitando o consenso gerado pelos alinhamentos de amplos setores envolvidos na luta contra o nazismo.

$\mathrm{Na}$ avaliação dos interesses estratégicos englobados por suas linhas de ação, o EB via a costa brasileira, na qual incluía a floresta amazônica, como um "vasto laboratório" e portanto uma prioridade primeira ${ }^{\mathrm{i}}$. A América Latina era vista como uma das mais importantes regiões etnogeográficas nas quais a pesquisa social desabrochava na época. Ainda que para o EB fosse uma área entre muitas, registram-se nos diários de seu coordenador Duncan Strong encontros regulares, e mesmo em horas inusitadas de fins de semana, nos quais se reunia frequentemente com os especialistas sobre América do Sul e seus índios ${ }^{\mathrm{ii}}$.

A organização governamental específica para lidar com a América do Sul foi o Office of the Coordinator of Inter-American Affairs (OCIAA), criado pelo Council of National Defense, em 16 de agosto de 1940, para responder à percepção do que o Departamento de Estado considerava uma ameaça massiva à segurança dos EUA. Este escritório foi estabelecido, essencialmente, para assistir o governo na preparação e coordenação de políticas para estabelecer as economias latino americanas e para "assegurar e defender a a influência dos EUA na região, e para combater o acesso do Eixo ao hemisfério, particularmente em esferas comerciais e culturais" (Cramer and Prutsch, 2006:786) $)^{\mathrm{iii}}$.

Quando os EUA, depois do bombardeio de Pearl Harbor (Dezembro de 1941), entraram na guerra, o Brasil - já que a almejada eqüidistância nas relações comerciais com as potências era tornou-se inescusável - declarou solidariedade ao governo dos EUA, unindo-se às forças aliadas contra o Eixo. Em troca, os EUA equiparam militarmente e industrialmente seu estratégico "bom vizinho". Esta aliança foi decisiva para quebrar, logo no início de 1942, o apoio argentino e chileno ao Eixo. Se com tal 
posicionamento brasileiro, tornou-se possível tratar de uma política de composição panamericana, em prol da qual se desenvolveram amplas estratégias de propaganda. Diversas personalidades, artistas e pessoas influentes visitaram o Brasil em uma verdadeira campanha deflagrada em tempo de guerra.

A entrada do Brasil na guerra era almejada principalmente por seu papel de fornecedor de matéria prima como ferro, manganês, e, sobretudo, borracha natural. A Rubber Development Coorporation foi criada para estimular produção de borracha imediatamente com os fins estritos voltados ao esforço de guerra. O principal objetivo era incrementar os lucros dos EUA na balança comercial internacional no sentido de promover sua segurança militar (Dean, 1987:88).

Rockefeller tinha uma interesse especial na Amazônia, tendo conduzido um projeto de saúde baseado em um acordo intergovernamental envolvendo os EUA e o Brasil. A Amazônia era vista como uma fronteira econômica favorável a programas de desenvolvimento que nasciam na arena internacional (Weinstein, 2007). Em tempos de guerra, Rockefeller investiu especialmente em programas relacionados à exploração da borracha. Esta política desenvolvimentista era um preâmbulo para a locação de agrupamentos locais considerados favoráveis para os investimentos em tempos de guerra fria, situando o Brasil como uma aliado mais favorável para a política de "boa vizinhança” que países da América Latina ou Caribe como Mèxico ou Cuba.

O National Research Council, criou em 1949 o Comitê de Antropologia Latino Americana, que antes da guerra, refletia um desejo expresso "de integrar pesquisa profissional com programas governamentais" iv. Um dos objetivos deste comitê foi a criação de uma lista de especialistas que incluía pessoal disponível para serviços como consultores especiais para situações de emergência, treinamento e pesquisa em atividades militares, e pesquisadores desenvolvendo estágios em áreas de interesse 
estratégico para os EUA.Duncan Strong contatou professores que lideravam a cena acadêmica para colaborar com este comitê. Eles enviaram listas de recomendações para a composição do "corpo de especialistas" do EB, os quais, integrados em comitês de áreas, eram recrutados pra serviço governamental.

Antropólogos engajados em tais comitês e organizações usaram seus conhecimentos academicamente adquiridos para práticas profissionais, o que não era tradicionalmente considerado como pesquisa de campo etnográfica.

\section{Nimuendaju e o campo científico: um informante privilegiado para a} ecologia cultural?

A leitura da correspondência de Steward mostra que no curso da organização do volume sobre Florestas Tropicais do Handbook of South American Indians Steward mudou sua visão sobre Nimuendaju e o papel das fontes etnográficas na prática científica. Inicialmente, Steward pensou Nimuendaju como um colaborador secundário, que necessitava ser ajudado por Lowie para publicar seus trabalhos sobre os grupos indígenas da Amazônia. Foi só após sua viagem ao Brasil, em fevereiro de 1942, quando encontrou pessoalmente com ele, que Steward se deu conta da significação antropológica do etnógrafo alemão naturalizado brasileiro. Como editor do Handbook, no entanto, impôs regras que Nimuendaju nunca aceitou inteiramente e a interação dos dois sempre foi muito tensa.

Steward corroborava a divisão hierárquica do trabalho que impregnava o habitus antropológico de seu tempo. Apesar de considerar Nimuendaju como um infomante chave, não considerava Nimuendaju como um teórico que pudesse apresentar a mesma contribuição que professores academicamente treinados como Robert Lowie, que Steward encarregara da introdução teórica do Hanbook . 
Essa visão hierárquica peculiar ao campo científico da época embebia também a abordagem de Nimuendaju, visto que ele também considerava como fontes secundárias informações fornecidas por Constant Tastevin, como se pode depreender da crítica de Nimuendaju ao artigo do missionário sobre os índios Mura (Tastevin, 1923). Nos originais que Nimuendaju enviou ao Handbook, depositados nos NAA, ele critica interpretações errôneas do missionário, que ele considera “completamente inadmissíveis”. No entanto, a tradução de seu artigo publicada no Handbook suprimiu sua crítica a Tastevin(.(Nimuendaju, 1948, 255)Apesar de sua crítica aos desacertos linguísticos de Tastevin, Nimuendaju usava fartamente, inclusive em seu celebrado mapa etnohistórico, informação coletada pelo missionário, não podendo deixar de reconhecer a contribuição histórica de seus registros para o conhecimento dos povos indígenas amazônicos.

Nimuendaju coletou, em colaboração com Lowie, informação sobre mitos nativos, depoimentos e objetos etnográficos. Tais artefatos eram dotados de significado cultural e Nimuendaju neles trabalhou não apenas como coletor e tradutor, mas também reelaborando-os em termos de um conhecimento antropológico acumulado previamente. Escrevendo sobre cada povo especifico com o qual interagiu, Nimuendaju criou quebracabeças etnográficos, revelando as significativas diferenças e similaridades entre traços culturais de diferentes povos, permitindo comparações antropológicas ${ }^{\mathrm{V}}$ Nos últimos dias de sua vida, dedicou-se à etnografia dos Ticuna, um único povo vivendo em uma situação convulsionada na fronteira entre o Brasil, o Peru e a Colômbia. Em 2002 interagi em pesquisa de campo que se lembravam da atuação de Nimuendaju como um ator que concorreu para a afirmação de sua identidade face a representantes da sociedade envolvente. 
As cartas de Lowie a Steward mostram que Nimuendaju reivindicava reconhecimento de sua contribuição como um autor. Juntamente com tal demanda de capital simbólico, expunha que necessitava remuneração material Nimuendaju não era empregado pelo Museu Goeldi e, portanto, dependida para sua subsistência de transações comerciais baseadas na avaliação financeira de seu trabalho.

Apresentando Nimuendaju como seu "co-worker", Lowie solicitou a Steward dinheiro para apoiar sua pesquisa de campo ${ }^{\text {vi }}$. No entanto, a Smithsonian apenas pagava a escrita de trabalhos avaliados estritamente pelo número de palavras. $\mathrm{O}$ editor considerava os colaboradores como fontes de informação, não os reconhecendo como comparáveis com os autores academicamente treinados para a profissão de antropólogos Segundo Steward, a Smithsonian tinha que enviar a conta para o HSAI ao congresso, como parte de uma apropriação geral para o projeto cujos objetivos eram traçados como estratégias da "política de boa vizinhança" com países da América do Sul. O projeto estava portanto fora da esfera limitada e das responsabilidades do Bureau of American Anthropology e seus gastos necessitavam seguir regras e caminhos burocráticos ${ }^{\mathrm{vii}}$. Em relatórios da Smithsonian, encontram-se demonstrativos de salários pagos a pesquisadores e professores, que tinham suas despesas de campo custeadas por orçamentos específicos. Isto não ocorreu com os colaboradores "voluntários", os quais, ainda que reconhecidos como importantes fontes de informação, não eram reconhecidos como iguais pelos profissionais academicamente treinados como antropólogos.

Lowie negociou com Steward uma remuneração para Nimuendaju, veiculando suas reivindicações de apoio para o seu trabalho. Não se tratava da venda de textos e artefatos coletados que custeassem as pesquisas e a subsistência do pesquisador e dos informantes, que caracterizavam a divisão do trabalho no campo antropológico, previamente, visto que na produção do Handbook as relações entre a fonte pagadora e 
os autores obedeciam a toda uma regulamentação que estipulava pagamento por palavras mediante contrato formal. Lowie informou que Nimuendaju levaria todo o um mês para preparar uma cópia de seu gigantesco mapa etnohistórico, com as mudanças e correções apontadas por Lowie ${ }^{\text {viii }}$. Segundo Lowie, Nimuendaju não impusera preço deixando a tarefa aos organizadores do volume.

Em sua carta, Nimuendaju informou que o apoio necessitado era para pagar seus informantes e suas despesas de subsistência enquanto o atendiam. Em outra carta, dirigida a Harold Shultz ${ }^{\text {ix }}$ ele afirmou que o melhor tempo para estudar (sic) os Ticuna era a estação das chuvas, quando eles não estavam trabalhando com a extração da borracha, agricultura e pesca, e estavam "livres" para acompanhá-lo. Em 1944 Nimuendaju acusa recebimento de sobre as miçangas enviadas por Eduardo Galvão, comentando que os Ticuna ficaram muito felizes em recebê-las e usá-las preparando pulseiras e colares para rituais de puberdade feminina. Nimuendaju tinha consciência do papel que desempenhava nas performances culturais, identificando-se a si mesmo com os povos indígenas perseguidos pelos patrões. Em várias $\operatorname{cartas}^{\mathrm{x}}$, Nimuendaju notou que era identificado por estes comerciantes como uma ameaça porque dada sua proximidade com os índios eles julgavam que ele os encorajava a desafiá-los. Entretanto, apesar de suas trocas simbólicas e culturais com os índios, reconhecendo suas habilidades como “artistas", "escultores" e "artesãos", Nimuendaju reificava a hierarquia imposta pela pesquisa antropológica, qualificando os Ticuna como "informantes".

A identificação de Nimuendaju com os povos discriminados não era unicamente uma circunstância com a interação com os índios durante suas pesquisas de campo. Ele não se considerava a si mesmo como um membro das elites brasileiras. Vivendo em Belém, ele vivia em relações maritais com uma lavadeira cujo registro de óbito a identificou como "parda". Esta mulher, depois da morte de Nimuendaju após vender seu 
arquivo para o Museu Nacional, dependia para sobreviver da ajuda financeira de pesquisadores do Museu Goeldi. As opiniões de quem o conheceram são muito controvertidas. Segundo relato do Sr Raimundo Nonato Brito, por mim entrevistado que trabalhou com menino de recados para Berringer \&Cia, firma que enviava a correspondência de Nimuendaju para outros estados do Brasil e para o exterior, ele sempre andava vestido com trajes de expedicionário, parecendo uma pessoa distante que apenas lidava com livros e ciência. Outros o consideravam um tipo não convencional, relacionado e círculos boêmios e freqüentador de prostíbulos pobres (Expedito Arnaud, i.p.).

Nimuendaju trabalhou com Lowie de 1935 a 1942, apoiado por recursos do Institute of Social Sciences da Universidade da Califórnia, sendo nos relatórios de Lowie considerado seu "assistente de pesquisa". No primeiro ano recebeu U\$700 para despesas com pesquisa de campo. De 1936 até 1941 recebeu U\$1.200 por ano. Em 1942, no entanto, este instituto apenas podia lhe fornecer $U \$ 300^{\mathrm{xi}}$. Ainda que a antropologia social não fosse o principal foco da Rockefeller Foundation (RF), esta instituição apoiara pesquisa antropológica desde os anos 1930s, fornecendo pequenas somas para pesquisa acadêmica nas universidades de Yale e Columbia. Lowie solicitou U\$500 à Fundação Rockefeller para suplementar os fundos para a viagem de Nimuendaju de 1942 ao alto Solimões ${ }^{\text {xii }}$. A carta de Lowie para a Rockefeller enfatizou os méritos do etnógrafo e explica porque ele necessita do dinheiro ${ }^{\text {xiii }}$.

Alfred Metraux e Carl Sauer também enviaram cartas de recomendação para a Rockefeller, tentando minimizar o fato que Nimuendaju não era um pesquisador formado academicamente. Sauer descreveu Nimuendaju "as a civilized European who can go native and experience these native cultures as a participant, and yet record them as a scientist" ..."an excellent craftsman doing a piece of work that no one else can do". 
Sauer também argument ou que o etnógrafo era "the old case of the individual scholar who finds a field to which he devotes his life", mencionando especificamente o "the old problem of individual research versus a research program into which workers are fitted. I don't know what the answer is administratively to this bothersome question of grantin-aid to the lone scholar, but until the foundations find this answer the opportunists, imitators, and apprentices have all the advantage over the men who make the contributions to knowledge". ${ }^{\text {iv }}$ Willit respondeu afirmando: "As to the problem of the lone scholar who has no institutional connections, I can only quote Kipling's poem 'No tangle's so tangled it cannot improve if the lover has brains " "xv .

A rede de apoio que Lowie e seus colegas tentavam prover à viagem de Nimuendaju realmente era um ninho emaranhado. Nimuendaju necessitava dinheiro para viajar para campo tão cedo possível porque necessitava finalizar suas observações de sua viagem de 1941. Como o comitê da Rockefeller decidiu fornecer o dinheiro através de instituições brasileiras, o Museu Nacional e o Museu Goeldi disputaram o papel de intermediário tendo em vista garantir a custódia de suas coleções etnológicas. Heloisa Alberto Torres, Diretora do Museu Nacional, era membro do Conselho de Fiscalização das Expedições Artísticas e Científicas Nacionais (CFE) e usou suas prerrogativas para tentar intermediar a transação. No entanto, em uma carta anexa à correspondência enviada por Metraux à Rockefeller, Nimuendaju respondeu enfaticamente que ele preferia que o dinheiro fosse enviado diretamente para ele através do Museu Goeldi, principalmente devido a condições de tempo, uma vez que a se o dinheiro fosse enviado antes ao Rio de Janeiro, ele teria que adiar sua viagem devido a condições metereológicas, visto que a estiagem dificultaria muito seus deslocamentos em campo e o impossibilitaria de chegar a certos lugares Ticuna que precisava visitar ${ }^{\mathrm{xvi}}$. 
Na carta a Willitz à a qual anexou a resposta a Nimuendaju Metraux expôs, a título confidencial, as complicadas teias da intriga, tentando explicar a rivalizade entre as instituições brasileiras e mostrando que a relutância da Rockefeleller em enviar o dinheiro diretamente ao museu de Belém iria privar Nimuendaju da dotação que ele necessitava e merecia. Metraux explicou que Nimendaju não iria manter as coleções para seu uso pessoal. Metraux também descreveu o perfil de Dona Heloisa, bem como seu ciúme das prerrogativas Museu Nacional considerando os laços de estima mantido por ela com relação a Nimuendaju, considerando o apreço à sua contribuição etnológica ${ }^{\mathrm{xvii}}$.

Em carta enviada por Dona Heloisa a Soper, o representante da Rockefeller no Brasil, ela concordou que o dinheiro poderia ser enviado a Belém. Entretanto, ela sugeriu uma proposta tentando conciliar o interesse das instituições. Sua aceitação que o dinheiro fosse enviado a Belém, estava condicionada à expectativa que parte dos artefatos coletados por Nimuendaju em viagem financiada pela Rockefeller fossem enviados ao Museu Nacional. O fato de ser Diretora do Museu Nacional e membro do CFE era explicitado como um fator de legitimidade da sua demanda. Concorria à justificativa a alusão ao seu sentimento de que Nimuendaju tinha um débito de lealdade a ela, por todo o apoio que ela vinha concedendo às pesquisas de campo dele ao longo de anos, ajudando-o tanto em assuntos antropológicos quanto pessoais “xviii .

Metraux anexou a sua carta a tradução de uma segunda missiva de Nimuendajú na qual ele mencionou seus percalços com o CFE e suas dúvidas no tocante a suas responsabilidades junto a tal conselho, visto que nas presentes viagens ele estava colaborando com instituições nacionais e não expedições privadas. A carta de Nimuendaju caracterizou como xenofóbicas as ações nacionalistas que visavam restringir sua pesquisa e cercear seu movimento. Entretanto, como confidenciou a 
Metraux, ele também pressentia que ambos os diretores do Museu Nacional e do Museu Goeldi estariam de seu lado em caso de qualquer objeção colocada ao seu trabalho pelo CFE ou outros grupos de interesse de âmbito local ou nacional ${ }^{\text {xix }}$.

Nimuendaju via Carlos Estevão e Dona Heloisa como seus melhores amigos no Brasil e assegurava sua submissão mediante laços de lealdade a ambas as instituições. Sua insistência em receber o dinheiro via Museu Goeldi devia-se a questões operacionais, desde que ele não trabalhava diretamente para o Museu Nacioal. Nimuendaju vivia em Belém e estava fisicamente mais próximo a Carlos Estevão que a Heloisa. Ele aceitou que os artefatos coletados em sua viagem pudessem ser divididos entre as duas instituições, contanto que fossem enviadas ao Museu Goeldi. A informação oficial enviada de Manaus pelo "delegado" Agnelo Bittencourt ao CFE é que Nimuendaju remeteu seu material de Manaus para o Museu Goedli, onde foi dividida entre esta instituição e o Museu Nacional. ${ }^{\mathrm{xx}}$.

Antes de encontrar o etnógrafo em Belém em 1942, Julian Steward reconheceu formalmente a extensa contribuição de Nimuendaju para o Handbook, estabelecida mediante contrato. Após o encontro, ambos entabularam correspondência sistemática, na qual manifestam tensões de pontos de vista. Nimuendaju discordou de Steward apontando a imprecisão das traduções para o inglês de seu trabalho sobre os índios Ticuna e os sumários arbitrários de suas descrições etnográficas de grupos indígenas particulares. Era difícil para Nimuendaju aceitar as regras quantitativas estabelecidas por contrato com a Smithsonian para a colaboração com o Handbook. Em carta a Metraux, que passou a intermediar a interação de Nimuendaju com o editor, Nimuendaju sustentou uma argumentação qualitativa, explicando sua demora em enviar sumários superficiais sobre índios desconhecidos, uma vez que estava imerso em um trabalho que considerava mais importante, a monografia sobre os índios Ticuna, 
resultante de sua colaboração com Lowie em Berkeley ${ }^{\text {xxi }}$ (publicada postumamente em 1952).

Seria uma simplificação considerar a aspiração de Nimuendaju de ser reconhecido como um autor meramente como uma reivindicação por recursos econômicos e recompensa material para sua produção para o Handbook. Nimuendaju preferia escrever sem remuneração para terminar sua monografia sobre os Ticuna que produzir sumários pagos. Respondendo às pressões de tempo de Steward, ele argumentou na citada carta de Metraux que ele preferia produzir um produto qualitativamente superior que muitos artigos incompletos. Seus argumentos mostram que ele acreditava que as regras do produtivismo institucional não podem sobrepujar o objetivo de qualidade científica inerente à tarefa antropológica. Visava qualidade cientifica apesar da demanda formal de quantidade de material publicado apressadamente atendendo a cronogramas burocráticos que minimizavam o peso da qualidade acadêmica.

\section{Conclusão: Fronteiras antropológicas e etnografia de fronteiras}

\section{indígenas}

- Curt Nimuendaju realizou etnografias extensivas sobre inúmeros povos pouco conhecidos tornando-se legendário no campo antropológico. A problemática da fronteira antropológica está subjacente ao exame relação de Nimuendaju como etnógrafo que lidou com o problema da situação de fronteira étnica, geográfica e econômica vivida pelos índios Ticuna. Inegavelmente sua interação com estes índios envolveu problemas de identidade, nacionalidade e etnicidade em fronteiras. Mas este trabalho extrapola estas questões, focalizando antes a produção de sua etnografia como 
uma prática fronteiriça que envolve fronteiras entre diferentes modos de fazer e pensar a disciplina tal como se configuram conforme diferentes nacionalidades.

De origem alemã e vivendo na Amazônia, a visão de Nimuendaju sobre a antropologia era forçosamente diferente da visão de seus interlocutores, notadamente Julian Steward, que trabalhava com recursos do Departamento de Estado dos Estados Unidos e deste modo estava envolvido com um projeto nacional expansionista em luta pela hegemonia política e cultural sobre os estudos americanistas.

O conhecimento antropológico produzido por Nimuendaju por longo tempo na "linha de frente", incorporou além do conhecimento dos próprios índios, o conhecimento produzido por um gênero especial de viajantes que durante a primeira metade do século XX registraram seu testemunho sobre as transformações históricas.

As fontes de recursos de tais viajantes baseavam-se na negociação de textos e artefatos que eram adquiridos pelos museus eram convertidos em bens culturais que rendiam fundos para quem os coletara. Estes fundos serviam como fonte de financiamento para as viagens de exploração. A criação de agências financiadoras que selecionavam os trabalhos a serem apoiados com base em uma avaliação hierárquica da qualidade da formação acadêmica e da produção científica e com a regulação do pagamento por meio de contratos formais deu uma nova forma à produção do conhecimento buscando regulamentar a negociação do valor das informações coletadas. Visando explicar a partir das metrópoles o conhecimento coletado alhures, reproduziusse, em meados do século $\mathrm{XX}$, a hierarquia entre teóricos de gabinete e viajantes coletores e a divisão de trabalho da exploração colonial, constituindo assim fronteiras sociais entre pesquisadores, com base em diferenças entre modos de produção de conhecimento em diferentes formações nacionais. 
Durante a Segunda Guerra Mundial, a pesquisa de campo na Amazônia não estava diretamente ligada ao esquema direto da intervenção de guerra, cujo foco estava na Alemanha nazista. Mas a Amazônia aparecia como uma importante região tanto do ponto de vista estratégico na política de boa vizinhança, quanto econômico, dada a necessidade imediata de incremento dos estoques de borracha, produto disponível nas seringueiras nativas da floresta tropical. A região representou uma espécie de "fronteira cultural" que também foi afetada pela guerra. Era vista como "a última fronteira", o local do "encontro de culturas", um lugar privilegiado para observar antropologicamente a interação de natureza e cultura. Também era um sítio propício para o simbolismo da integração pan americana, mais interessante para os planos de desenvolvimento que o México, muito revolucionário para o liberalismo expansionista. A ideia que os índios brasileiros eram uma massa de manobra para o desenvolvimento político era o reverso da imagem sonhada por americanistas forasteiros de que estes povos viveriam "autenticamente" em "estado de natureza" A noção de desenvolvimento implicava a representação de assimilação e integração dos índios que, integrados ou assimilados não poderiam mais viver em "estado de natureza". Pode se dizer, para finalizar, que Nimuendaju produziu sua monografia sobre as fronteiras Ticuna em uma situação de fronteira antropológica.

\section{BIBLIOGRAFIA}

Bennet, Wendell Clark

1947 The Ethnogeographic Board”. "Smithsonian Miscelanean Collections. Vol. 107 n. 1, pag V-130.Washington, Smithsonian Institution. 
Cramer, G \& Prutsch, U.

2006 Nelson Rockefeller's Office of Inter-American Affairs and Record Group 229. Hispanic American Review, vol. 86 (4): 785-806.

Dean, Warren 1987 - "The battle for rubber, 194-1945". Brazil and the struggle for Rubber. A Study in Environmental History. Cambridge/New York, Cambridge University Press, pp. 87107

Elias, Norbert

1982 "Scientific Establishments". Scientific Establishments and Hierarchies (Ed.: Norbert Elias, Herminio Martins and Richard Whitley). Dordrecht, Boston and London, D. Reidel Publishing Company, p. 3-70.

Nugent, David

2008 - "Social Science Knowledge and military intelligence: global conflict, territorial control and the birth of area studies during WW II" Anuário Antropológico 2006, Brasília/Rio deJaneiro, UNB/Tempo Brasileiro, pp. 33-68

Pels, Peter 
1997 - "The Anthropology of Colonialism: Culture, History and the Emergence of Western Governmentality" Annual Review of Anthropology 26, 163-183

2008 - "What was anthropology learned from the anthropology of colonialism?". Social Anthropology/ Anthropologie Sociale 16, 3 280-299.

Ranelagh, John

1986 - The Agency: The Rise and Decline of the CIA. New York, Touchstone.

Simmel, Georg

1968 - "On the concept and tragedy of culture" in: Conflict in Modern Culture and Other Essays. New York, Teachers College Press, pp. 27-46

Steward, Julian H.

1941 - "Handbook of South American Indians" - America Indigena, Vol.1, n. 1. Mexico, Insttituto Indigenista Interamericano, pp. 47-50.

1948 Handbook of South American Indians (ed). Volume 3. The Tropical Forest Tribes. Washington DC, Smithsonian Institution.

1955 - Theory of Cuture Change. Illinois, University of Illinois Press.

Stocking, George

1976 "Introduction: Thoughts toward a History of the Interwar Years. In:American Anthropology, 1921-1945 (ed.: G. H. Stocking Jr),Lincoln and London, University of Nebrasca Press, 1-74

Weinstein, Barbara 
2007 “Modernidade Tropical: visões norte-americanas da Amazônia nas vésperas da

Guerra Fria”. Revista do IEB, n.45 p. 153-176 set 2007.

\footnotetext{
${ }^{\mathrm{i}}$ Carta de Steward a Lowie, 10 de setembro de 1942, NAA/ISA

ii NAA, BOX 49, Journal of WM Duncan Strong as Director of the Ethnogeographic Board.

iii President Roosevelt apontou Nelson Rockefeller como Coordinator of Inter-American Affairs ao mesmo tempo que apontou Willian Donovan como Coordinator of Information. O Office of Strategic Services, criado 1942, tornou-se um embrião da CIA (Central Intelligence Agency of USA) (Ranelagh, 1986).
}

iv Records of BAE, Series 4, Miscellaneous Administrative Files (1948-1965- Box 289), Bureau of American Ethnology, Memorandum re: A guide to the Survey of Foreign Liaison Activities of the Research groups within the Federal Government (9 páginas).

${ }^{v}$ Como um exemplo de tais quebra-cabeças, podemos indicar a correlação entre mitos Sherente, Ticuna and Apopokuva e sua observação dos fenômenos do céu, que permitem comparações entre costelações relacionadas a diferentes cosmovisões indígenas.

${ }^{\text {vi }}$ Carta de Lowie a Steward, 16 de Outubro de 1940.

vii Carta de Steward para Lowie 21 de Junho de 1939

viii Steward adquiriu na viagem de 1942o mapa etnohistórico de Ninuendaju, É curioso que neste mesmo ano de 1942 o Comitee of Inter American Affairs produziu um mapa identificando locais de potencial trabalho indígena para a extração de borracha, utilizando as fontes etnohistóricas compulsadas por Nimuendaju.

${ }^{i x}$ May, 31, 1945.Arquivo Nimuendaju, Museu Nacional/UFRJ.

${ }^{x}$ Carta a Heloisa .Alberto Torres 3 de agosto de 1941.

xi "Application for research grant form funds of the Institute of Social Sciences, University of California" signed by Robert Lowie on March 6, 1941 Documento encontrado em uma pasta confiada por Lowie à custódia de John Rowe(JRA). Agradeço Patricia Lyon pelo acesso à documentação.

xii "Report on Sr. Curt Nimuendajú's work since 1938", Assinado por Lowie em 2 de Fevereiro de 1942, 4 páginas (JRA).

xiii RA RG 1.1Series 305, Box 54, Folder 289.

${ }^{\text {xiv }}$ De Carl Sauer para J.H. Willitz 29 de Nov. de 1941, RA ibid.

${ }^{x v}$ De Willit para Sauer, 3 de dezembro de 1941, RA, ibid.

xvi ... (Carta de Nimuendaju anexa à carta de Metraux a Willitz, ibid)

xvii de Metraux para Willits, 14 de maio de 1942.(RA, ibid).

xviii "Carta de Dona Heloisa para Sopper, , 5 de fevereiro de 1942, RA.

xix “... 1 de março, 1942, RA, Segundo anexo à carta de Metraux para Willits, 14 de maio de 1942.

${ }^{x x} 11$ de outubro de 1942 (AHC/MAST).

xxi . Nimuendaju recebeu um total of $\$ 400$ para o conjunto de seu trabalho como atestado pelo recibo enviado para Julian Steward (Belém do Pará, 6 de novembro de 1943, NAA/RHSAI) 